\title{
Epitaxial growth of complex oxide films: Role of surface reconstructions
}

\author{
Michele Riva $\odot,{ }^{*}$ Giada Franceschi $\odot$, Michael Schmid, and Ulrike Diebold \\ Institute of Applied Physics, TU Wien, Wiedner Hauptstraße 8-10/E134, 1040 Wien, Austria
}

(Received 3 July 2019; revised manuscript received 2 August 2019; published 29 October 2019)

\begin{abstract}
Achieving atomically flat and stoichiometric films of complex multicomponent oxides is crucial to integrating these materials in both established and emerging technologies. While pulsed laser deposition (PLD) can in principle produce these high-quality films, growth experiments often result in unsatisfactory morphologies with rough surfaces and nonstoichiometric compositions. To understand the cause, the growth needs to be followed at an atomic level from its early stages as a function of the growth conditions. By combining PLD with atomically resolved scanning tunneling microscopy, as well as surface spectroscopic and diffraction techniques, we address the origin of surface roughening in $\mathrm{SrTiO}_{3}(110)$ homoepitaxial films and pinpoint optimal growth conditions. We highlight the importance of surface reconstructions at all stages of growth: The different sticking on coexisting surface structures is responsible for the roughening of $\mathrm{SrTiO}_{3}(110)$ films and affects their stoichiometry.
\end{abstract}

DOI: 10.1103/PhysRevResearch.1.033059

\section{INTRODUCTION}

Future high-performance electronic devices will rely on the coupling of multicomponent complex oxides in controlled thin-film heterostructures, where unexplored functionalities can be achieved by coupling lattice, electronic, charge, and spin degrees of freedom at well-defined interfaces [1-9]. The strict standards for stoichiometry and interface sharpness required for epitaxial heterostructures $[6,10]$ can in principle be met by pulsed laser deposition (PLD). In practice, achieving ideal films of complex oxides with PLD is not a trivial task. In fact, the film stoichiometry is affected by a multitude of PLD parameters, including, but not limited to, laser fluence, composition and pressure of the background gas, and substrate temperature [11-13]. For example, studies on the prototypical complex oxide $\mathrm{SrTiO}_{3}$ have shown that ideal compositions are only achieved with fine tuning of the fluence of the UV laser $[14,15]$. Moreover, the growth of complex-oxide thin films often results in rough or nanostructured surface morphologies.

A fundamental understanding of the mechanisms underlying nonstoichiometric growth and surface roughening is needed to achieve the desired high-quality films. The atomicscale details of the surface structure of the substrate might play a key role: Early studies on metals and semiconductors document the influence of the substrate reconstruction on the film morphology [16,17]. For more complex materials the literature on this topic is scarce, but where available it supports the important role of the substrate reconstruction for the film properties [18,19]. As a consequence, the reproducibility of

\footnotetext{
*Corresponding author: riva@iap.tuwien.ac.at

Published by the American Physical Society under the terms of the Creative Commons Attribution 4.0 International license. Further distribution of this work must maintain attribution to the author(s) and the published article's title, journal citation, and DOI.
}

a specific film morphology might rest on a sufficiently careful substrate preparation. In addition, the film morphologies might be affected by the choice of PLD parameters and by the nonstoichiometries that are consequently introduced.

By combining PLD growth with scanning tunneling microscopy (STM) [12], we address the atomic-scale origin of surface roughening in $\mathrm{SrTiO}_{3}(110)$ homoepitaxial films. We follow the growth as a function of the laser fluence, which systematically introduces nonstoichiometry. By growing homoepitaxial films we exclude that any of the observed phenomena arise from substrate-induced strain.

All three low-index surfaces of $\mathrm{SrTiO}_{3}$ adjust their surface structure in response to a change in surface stoichiometry [20-29]. We opt for the (110) orientation because of the reproducibility and detailed knowledge of its surface reconstructions, some of which are reported for reference in the phase diagram of Fig. 1 [20,30]. These reconstructions are $\mathrm{TiO}_{x}$ terminated, and form to compensate for the intrinsic polar instability of the bulk-truncated (110) surface [31]. Every reconstruction is an equilibrium phase, and monophase samples can be prepared for each of them. The $(n \times 1)$ reconstructions [Figs. 1(a)-1(c)] consist of a layer of corner-sharing, tetrahedrally coordinated $\mathrm{TiO}_{4}$ units lying on a bulk-truncated $(\mathrm{SrTiO})^{4+}$ plane [28]. The $(2 \times 4)$ structure [Fig. 1(d)] belongs instead to the $(l \times m)$ family, consisting of a double layer of octahedrally coordinated corner- and edge-sharing $\mathrm{TiO}_{6}$ units [32]. Importantly, the surface structure evolves along the phase diagram for submonolayer changes in the $\mathrm{Sr}$ and $\mathrm{Ti}$ composition in the near-surface region (bottom of Fig. 1) [20]. This has strong implications for the growth of $\mathrm{SrTiO}_{3}(110)$ films: Under nonstoichiometric conditions, the excess components float to the surface, changing its composition. Thus, the surface of a film changes its structure during nonstoichiometric growth and evolves along the surface phase diagram [20].

By measuring the change in the film surface structure via STM, we quantify the deposited cation imbalance as a 


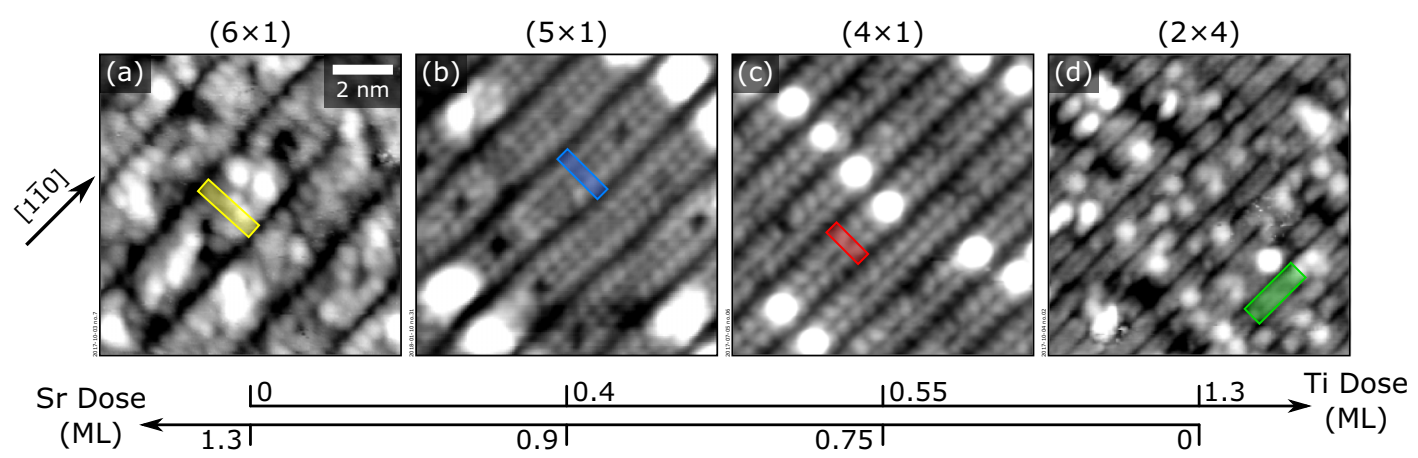

FIG. 1. Surface phase diagram of $\mathrm{SrTiO}_{3}(110)$. STM images $\left(10 \times 10 \mathrm{~nm}^{2}\right)$ of the $(n \times 1)(n=4,5,6)$ and the $(2 \times 4)$ reconstructions of $\mathrm{SrTiO}_{3}(110)$. The surface unit cell of each reconstruction is overlaid on the corresponding STM image. These phases are equilibrium structures governed by the near-surface stoichiometry: Submonolayer deposition (arrows at the bottom) of Sr or Ti, followed by high-temperature annealing in $\mathrm{O}_{2}$ ambient, allows to move continuously and reversibly between the surface structures. Here one monolayer (ML) corresponds to the number of $\mathrm{Sr}$ (or Ti) sites in a $(\mathrm{SrTiO})^{4+}$ plane of $\mathrm{SrTiO}_{3}(110)$, i.e., $1 \mathrm{ML}=4.64 \times 10^{14}$ atoms $/ \mathrm{cm}^{2}[20]$. STM parameters: $(\mathrm{a}) V_{\mathrm{b}}=+1.96 \mathrm{~V}$ and $I_{\mathrm{t}}=40 \mathrm{pA}$; (b) $V_{\mathrm{b}}=+0.88 \mathrm{~V}$ and $I_{\mathrm{t}}=40 \mathrm{pA}$; (c) $V_{\mathrm{b}}=+1.4 \mathrm{~V}$ and $I_{\mathrm{t}}=60 \mathrm{pA}$; (d) $V_{\mathrm{b}}=+2.0 \mathrm{~V}$ and $I_{\mathrm{t}}=50 \mathrm{pA}$.

function of the fluence of the ultraviolet (UV) laser [20]. We find that low laser fluences result in Sr-enriched films, consistently with literature reports [14,15,33]; instead, above a certain threshold, films grow close to stoichiometric or slightly Ti rich, in agreement with some previous results [33], but not as strongly Ti rich as observed elsewhere [14,15].

Besides allowing us to measure the cation nonstoichiometry via the change of the surface reconstruction, STM provides precise information about the atomic-scale morphology of our films: Low-fluence (Sr-rich) films always retain atomically flat surfaces, while rough surfaces are obtained at high fluences, where pits with a depth of a few layers appear. We unveil the origin of the pits: They form when new surface structures develop during growth that have lower surface sticking than surrounding areas. Ideal film morphologies and stoichiometries are achieved when the surface reconstruction is controlled at each stage of the growth.

\section{EXPERIMENTAL METHODS}

The growth experiments were performed in an ultrahigh vacuum (UHV) chamber with a base pressure below $4 \times 10^{-10}$ mbar after bakeout. It is equipped for high-pressure and high-temperature PLD growth (up to $1200^{\circ} \mathrm{C}$ ) [12]. During the growth, $\mathrm{O}_{2}(6 \mathrm{~N}$ purity) is dosed into the chamber via a leak valve, and a doubly differentially pumped TorrRHEED gun (Staib Instruments $\mathrm{GmbH}, 35 \mathrm{keV}$ beam energy, up to 1 mbar) is used to monitor reflection high-energy electron diffraction (RHEED) oscillations. The substrate is heated by a collimated continuous-wave infrared laser $(980 \mathrm{~nm})$ directed on the back of the sample through a hole in the sample plate. A small transfer chamber attached to the PLD chamber provides the interface to a connected surface science system. This UHV chamber has a base pressure below $5 \times 10^{-11}$ mbar and is equipped for STM (SPECS Aarhus STM 150), low-energy electron diffraction (LEED) (Omicron), and x-ray photoelectron spectroscopy (XPS) (nonmonochromatic dual-anode $\mathrm{Mg} / \mathrm{Al} K \alpha$ source, SPECS Phoibos 100 analyzer, normal emission).

The films were grown by PLD on Nb-doped (unless otherwise indicated) $\mathrm{SrTiO}_{3}(110)$ single crystals (CrysTec $\mathrm{GmbH}$,
0.5 wt. $\% \mathrm{Nb}_{2} \mathrm{O}_{5}, 5 \times 5 \times 0.5 \mathrm{~mm}^{3}$, one-side polished, miscut less than $0.3^{\circ}$ ). $\mathrm{SrTiO}_{3}(110)$ single crystals (also $\mathrm{Nb}$ doped, unless otherwise indicated) were used as targets (target-tosubstrate distance of $55 \mathrm{~mm}$, laser impingement on the target at $45^{\circ}$ with respect to its surface). Unless otherwise noted, deposition was performed at an $\mathrm{O}_{2}$ pressure of $6 \times 10^{-6} \mathrm{mbar}$, with an UV, KrF excimer laser (Coherent Compex Pro 201, $248 \mathrm{~nm}$ ), a repetition rate of $1 \mathrm{~Hz}$, sample temperature of $750^{\circ} \mathrm{C}$, and laser fluences between 1.3 and $3.9 \mathrm{~J} / \mathrm{cm}^{2}$. The choice of $n$ doping comes with the advantage that the equilibrium concentration of oxygen vacancies in $\mathrm{SrTiO}_{3}$ is negligible at our experimental conditions [34]. Therefore, only cation nonstoichiometries should be taken into account. Each deposition was followed by $10 \mathrm{~min}$ of postannealing at the growth conditions such that the RHEED intensity was fully relaxed [12]. This also ensures that any transient nonequilibrium of the concentration of oxygen vacancies is compensated [34,35]. The thickness of the deposited films was evaluated from the periodicity of intensity oscillations of the specular RHEED beam. Before growth, each substrate was prepared to exhibit a coexistence of $(4 \times 1) /(5 \times 1)$ surface reconstructions with $60-95 \%$ of the area showing a $(4 \times 1)$ structure. Shortly before deposition, a fresh, newly polished area of the $\mathrm{SrTiO}_{3}$ target was preablated at the same parameters that were later used for the growth by scanning the target in the UV laser beam, such that each spot was hit at least ten times. During this preablation the pristine sample was kept in a separate chamber. The energy of the UV laser (measured outside the PLD chamber with a through-chamber setup) was adjusted via a programmable attenuator (Coherent) shortly before preablation, as well as before growth. The effective fluence was computed by rescaling the laser energy to the size of the ablated spot on the target, as measured ex situ with an optical microscope [12].

The as-received samples were sonicated in heated neutral detergent (3\% Extran ${ }^{\circledR}$ MA02 diluted in ultrapure water, $2 \times 20 \mathrm{~min}$ ) and ultrapure water (milli- $\mathrm{Q}^{\mathrm{TM}}, 10 \mathrm{~min}$ ) to remove residues from mechanical polishing. Subsequent boiling for $10 \mathrm{~min}$ in milli- $\mathrm{Q}^{\mathrm{TM}}$ water removed the $\mathrm{CaO}$ contamination that we otherwise observe on some of the out-of-the box samples (likely originating from the polishing procedure). The 
samples were then mounted on a $\mathrm{HNO}_{3}$-cleaned Nicrofer ${ }^{\circledR}$ (high-temperature- and oxidation-resistant alloy) sample plate with Nicrofer clips (spot welded onto the plate). Once inserted in the UHV system, each sample was cleaned via (typically three) $\mathrm{Ar}^{+}$-sputtering-annealing cycles $\left(6 \times 10^{-6}\right.$ mbar $\mathrm{Ar}$, $1 \mathrm{keV}, 5-10 \mu \mathrm{A}, 10 \mathrm{~min}$, followed by $1 \mathrm{~h}$ at $1000^{\circ} \mathrm{C}$, $6 \times 10^{-6}$ mbar $\mathrm{O}_{2}$ ). The surface was then prepared to exhibit the desired reconstruction by adjusting the surface stoichiometry via submonolayer deposition of $\mathrm{Sr}$ [via molecular-beam epitaxy (MBE)] [36] or $\mathrm{TiO}_{2}$ (via PLD) [35], followed by annealing in oxygen $\left(30 \mathrm{~min}, 1000^{\circ} \mathrm{C}, 6 \times 10^{-6} \mathrm{mbar}_{2}\right)$. The quality of the pristine substrate (flatness, cleanliness, and correct reconstruction) was checked prior to deposition by XPS, LEED, and STM. All temperatures were measured via an optical pyrometer (IMPAC) aimed at the sample surface.

STM measurements were performed at constant current with homemade, electrochemically etched $\mathrm{W}$ tips. The tips were prepared in situ by Ar sputtering (1 $\mu \mathrm{A}, 30 \mathrm{~min})$. When needed to improve image resolution, voltage (up to $10 \mathrm{~V}$ ) or current pulses (up to $10 \mathrm{nA}$ ) were applied while scanning the measured samples. Positive bias voltages correspond to tunneling into the empty states of the sample. Error bars reported throughout the text correspond to $99 \%$ confidence intervals, as derived from the statistical evaluation of several (20-40) STM images [20]. These STM images were acquired at different spots on the sample, and the uniformity of the surface reconstruction in the area probed by STM was assessed by moving the sample in the LEED electron beam.

\section{RESULTS AND DISCUSSION}

\section{A. Laser fluence and nonstoichiometry in $\mathrm{SrTiO}_{3}(110)$ films}

We have shown in previous works that STM allows one to precisely quantify the deposited cation imbalance (here tuned with the laser fluence) during homoepitaxy on $\mathrm{SrTiO}_{3}(110)$, by carefully measuring the corresponding change in the surface structure [20]. Because the nonstoichiometry segregates to the surface during $\mathrm{SrTiO}_{3}(110)$ deposition [20], the excess material accumulates at the surface during growth. Thus, films of different thickness can be compared by measuring the nonstoichiometries introduced "per layer" (obtained by dividing the measured surface stoichiometry change by the number of layers). Figure 2 collects the nonstoichiometries per layer of various films (thickness between 7 and 52 layers, i.e., approximately equal to $1.7-14.3 \mathrm{~nm}$ ) that were introduced with different laser fluences; positive (negative) values stand for $\mathrm{Sr}$ (Ti) enrichment. We distinguish between two regimes, $\mathrm{A}$ and B. Fluences below $2.6 \mathrm{~J} / \mathrm{cm}^{2}$ (regime A) produce Sr enrichments, consistent with previous observations during homoepitaxial growth on $\mathrm{SrTiO}_{3}(001)$ [14,15,33]. Higher fluences (regime B) deliver slight Ti enrichments or almost-ideal stoichiometry, in accordance with the work of Dam et al. [33], but at odds with the pronounced Ti-enrichment documented elsewhere [14,15]. Below we argue that the deposition flux is actually (at least slightly) Ti rich above a certain threshold, as expected from literature findings on $\mathrm{SrTiO}_{3}(001)$ films $[14,15,33]$, but that the details of the (110) surface reconstruction and their sticking properties determine the quasi-ideal

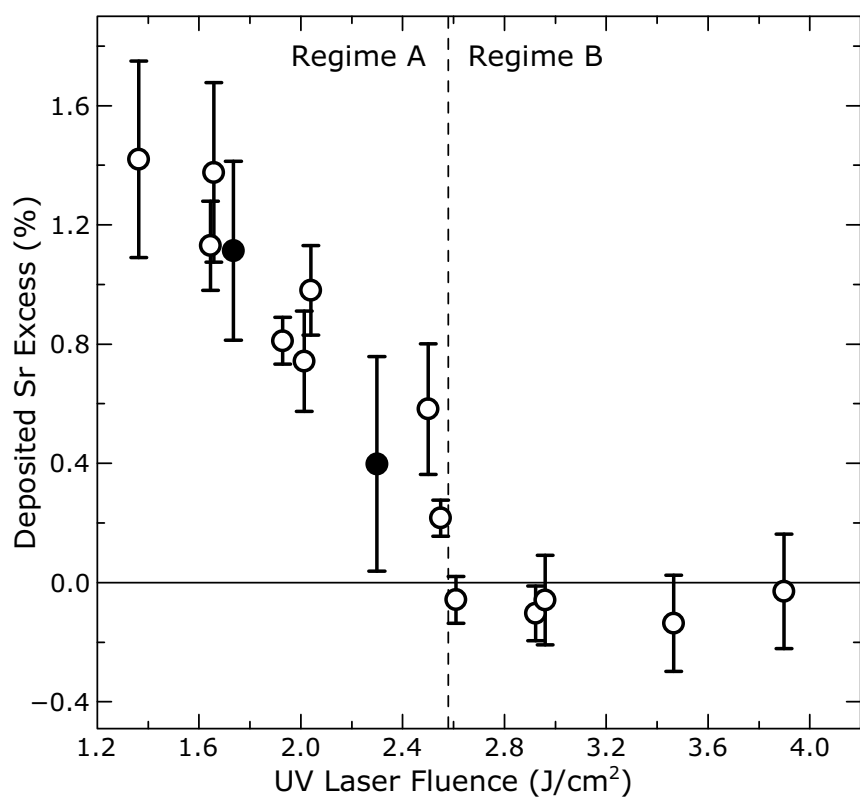

FIG. 2. Cation nonstoichiometry per deposited layer in homoepitaxial $\mathrm{SrTiO}_{3}(110)$ films as a function of the laser fluence. Open circles correspond to the homoepitaxial growth of Nb-doped $\mathrm{SrTiO}_{3}$ on $\mathrm{Nb}$-doped $\mathrm{SrTiO}_{3}(110)$ substrates, while full circles refer to the growth of undoped $\mathrm{SrTiO}_{3}$ films on undoped $\mathrm{SrTiO}_{3}(110)$. The nonstoichiometries are measured with STM, by relating the change in the surface structure during growth to the corresponding composition change via the phase diagram of Fig. 1. The graph expresses the $\mathrm{Sr}$ excess introduced per deposited layer, obtained by dividing the measured surface stoichiometry changes by the film thickness (expressed in number of layers) [20]. Below a threshold (dashed vertical line), decreasing fluences result in $\mathrm{Sr}$ enrichment (regime A), while higher fluences (regime B) produce slight Ti enrichment or close-to-ideal compositions.

composition observed. We also show that regimes $\mathrm{A}$ and $\mathrm{B}$ produce films with distinctive surface morphologies.

We note that the values of the threshold fluence reported in the literature are quite scattered (ranging from 0.3 to $\left.2.3 \mathrm{~J} / \mathrm{cm}^{2}[14,15,33,37]\right)$ and differ somewhat from the value of $2.6 \mathrm{~J} / \mathrm{cm}^{2}$ that we obtained (Fig. 2). We can think of at least three reasons. (i) In addition to the nominal value of the laser fluence, several other factors can affect the introduced nonstoichiometries, including $\mathrm{O}_{2}$ background pressure, target-to-substrate distance and alignment, laser spot (dimension, uniformity, and shape), aging of the UV laser gas (we found that an "old" excimer laser gas systematically results in Sr-richer films), preablation of the target (the target should be sufficiently preablated at the same conditions used during growth), choice of the ablation area (when picked too close to a previously ablated area, redeposited material from the first area can affect the results), and unreliable measurement of the laser fluence. (ii) The various designs of commercial PLD chambers and the lack of accepted methods to measure many relevant parameters can complicate the consistency of the quantitative data from laboratory to laboratory $[12,15]$. (iii) The specific details of the surface structures of $\mathrm{SrTiO}_{3}(001)$ can affect the growth and consequently the introduced nonstoichiometries differently than on our $\mathrm{SrTiO}_{3}(110)$ films. It 

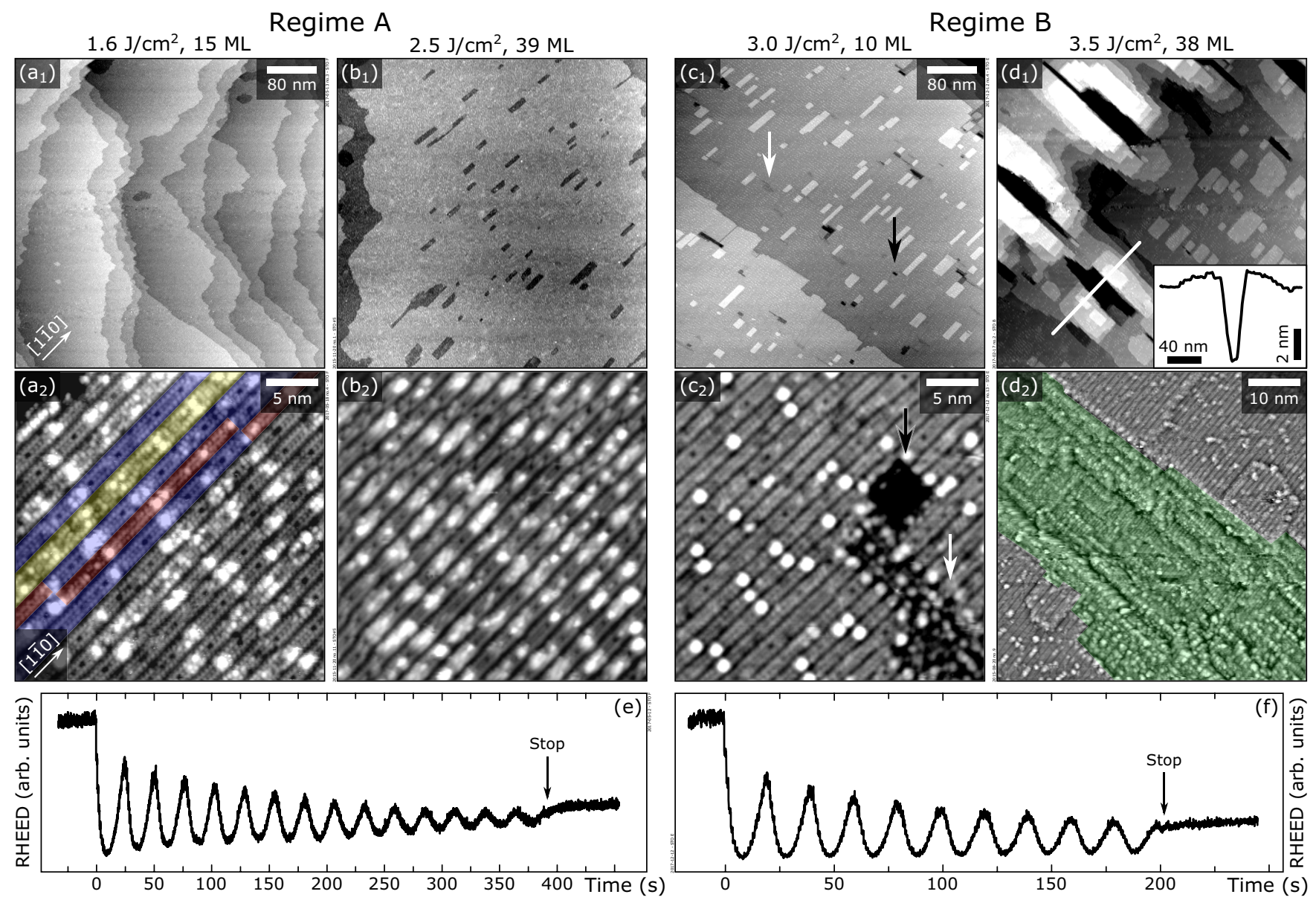

FIG. 3. Effect of nonstoichiometric deposition conditions on the surface morphology of $\mathrm{SrTiO}_{3}(110)$ films. STM images and RHEED intensity oscillations of representative, nonstoichiometric homoepitaxial $\mathrm{SrTiO}_{3}(110)$ films, obtained at different laser fluences (increasing from left to right). (a) and (b) Sr-rich films exhibit atomically flat surfaces with a mixture of $(n \times 1)$ lines. In panel $\left(\mathrm{a}_{2}\right)$, yellow, blue, and red areas mark $(6 \times 1)-,(5 \times 1)$-, and $(4 \times 1)$-reconstructed areas, respectively; in panel $\left(b_{2}\right)$, the surface consists almost exclusively of $(5 \times 1)$ lines. (c) Slight Ti enrichment triggers the formation of small $(2 \times 4)$ patches (white arrows) that interrupt $(n \times 1)$ terraces. (d) Higher fluences cause the appearance of few-layers-deep pits that are $(2 \times 4)$ reconstructed. A representative pit is shown in green in the high-pass filtered image in panel $\left(\mathrm{d}_{2}\right)$ from a 20-layers-thick film grown at $2.55 \mathrm{~J} / \mathrm{cm}^{2}$. In the inset of panel $\left(\mathrm{d}_{1}\right)$, the STM profile of one of the pits is shown. The precursors of the pits are indicated by black arrows in panel (c). (e) and (f) Specular-spot RHEED intensity oscillations of films $\left(a_{1}\right)$ and $\left(c_{1}\right)$ showing a behavior that is representative of films grown in regimes A and B, respectively. The STM parameters are $\left(\mathrm{a}_{1}\right) V_{\mathrm{b}}=+1.96 \mathrm{~V}$ and $I_{\mathrm{t}}=280 \mathrm{pA},\left(\mathrm{a}_{2}\right) V_{\mathrm{b}}=+1.79 \mathrm{~V}$ and $I_{\mathrm{t}}=30 \mathrm{pA},\left(\mathrm{b}_{1}\right) V_{\mathrm{b}}=+2.2 \mathrm{~V}$ and $I_{\mathrm{t}}=570 \mathrm{pA},\left(\mathrm{b}_{2}\right) V_{\mathrm{b}}=+1.1 \mathrm{~V}$ and $I_{\mathrm{t}}=170 \mathrm{pA},\left(\mathrm{c}_{1}\right) V_{\mathrm{b}}=+1.49 \mathrm{~V}$ and $I_{\mathrm{t}}=390 \mathrm{pA},\left(\mathrm{c}_{2}\right) V_{\mathrm{b}}=+1.63 \mathrm{~V}$ and $I_{\mathrm{t}}=40 \mathrm{pA},\left(\mathrm{d}_{1}\right) V_{\mathrm{b}}=+2.1 \mathrm{~V}$ and $I_{\mathrm{t}}=580 \mathrm{pA}$, and $\left(\mathrm{d}_{2}\right) V_{\mathrm{b}}=+2.2 \mathrm{~V}$ and $I_{\mathrm{t}}=360 \mathrm{pA}$.

is worth noting that quantitatively similar results are obtained if undoped $\mathrm{SrTiO}_{3}$ films are grown on undoped $\mathrm{SrTiO}_{3}(110)$ substrates (full symbols in Fig. 2), suggesting a minor role of the (possible) oxygen nonstoichiometry on the cation composition of the deposited films.

\section{B. Nonstoichiometry and surface morphology}

Our method has a high sensitivity to the cation imbalance introduced during growth (one order of magnitude better than the detection limit of 1-2\% typical of customary techniques) [20]. This allows us to precisely investigate the influence of nonstoichiometry on the film morphology. Figure 3 shows the surface morphology (top row) and the surface structure (middle row) of several representative $\mathrm{SrTiO}_{3}(110)$ films with different nonstoichiometries, obtained at increasing fluence from left to right. Regimes A and B (cf. Fig. 2) produce markedly different surface morphologies. Films belonging to regime A tend to retain flat surfaces [Figs. 3(a $\left.a_{1}\right)$ and $3\left(b_{1}\right)$ ], while high fluences (regime $B$ ) induce the development of pits a few layers deep [dark in Fig. $3\left(\mathrm{~d}_{1}\right)$ ] that get deeper, wider, and more numerous as the growth proceeds; high fluences are detrimental for the surface quality.

A closer look at the changes occurring at the atomic scale (middle row of Fig. 3) is of primary importance to understand the origin of the morphological roughening, as discussed below. We note that each substrate was initially prepared to exhibit a comparable surface to ensure a reproducible starting point: The surface always showed a mixture of interwoven $(4 \times 1)$ and $(5 \times 1)$ lines with a well-defined ratio [between $60 \%$ and $95 \%(4 \times 1)$ coverage]. Low-fluence (Sr-rich) films always retain a mixture of $(n \times 1)$ lines [Figs. $3\left(a_{2}\right)$ and $3\left(b_{2}\right)$ ]. A small Sr excess $\left[\right.$ Fig. $\left.3\left(b_{2}\right)\right]$ shifts the initially $(4 \times 1) /(5 \times 1)$ surface to almost exclusively $(5 \times 1)$ lines; the $\mathrm{Sr}$ excess is 
quantified by determining the change in the number of $(5 \times 1)$ lines as a result of the growth [20]. Larger Sr enrichments [Fig. $3\left(a_{2}\right)$ ] drive the initial $(4 \times 1) /(5 \times 1)$ surface to a mixed $(5 \times 1) /(6 \times 1)$ composition [the simultaneous presence of few $(4 \times 1)$ lines is assigned to an imperfect equilibration of the surface]; there the same concept can be applied for the evaluation of the Sr excess [20].

For fluences slightly above the threshold, a new, Ti-rich $(2 \times 4)$ phase appears on the $(4 \times 1) /(5 \times 1)$ terraces [see the small patches appearing darker in Figs. $3\left(\mathrm{c}_{1}\right)$ and $3\left(\mathrm{c}_{2}\right)$, some of which are indicated with arrows]. The formation of this Tirich reconstruction is consistent with the expected Ti enrichment of the flux at these conditions. At larger fluences and/or for thicker films, pits develop on the surface [Figs. $3\left(d_{1}\right)$ and $3\left(\mathrm{~d}_{2}\right)$ ]. Since similar pits were also observed during Tienriched MBE growth of $\mathrm{SrTiO}_{3}(110)$ [38], we can exclude that their formation is caused by possible sputter damage induced by the higher laser fluences employed in this regime. The large depth of 22 layers of the pits in Fig. $3\left(\mathrm{~d}_{1}\right)$, along with their narrow width [see the pit profile in the inset of Fig. $3\left(\mathrm{~d}_{1}\right)$ ], hindered obtaining reproducible atomic resolution at their base. Shallower pits enabled us to resolve that their bottom is $(2 \times 4)$ reconstructed; this is seen, for example, in Fig. $3\left(d_{2}\right)$, which displays the high-pass-filtered image of a nine-layers-deep pit obtained on a different film $[(2 \times 4)$ areas are shaded in green]. In general, LEED patterns acquired on high-fluence films (i.e., with pits) consistently display a $(2 \times 4)$ periodicity in addition to the $(4 \times 1) /(5 \times 1)$ periodicity associated with the $(n \times 1)$ terraces (not shown). Another representative feature of the pits is visible in Fig. $3\left(\mathrm{~d}_{2}\right)$ : rims of $(n \times 1)$ islands elongated along the [110] direction that decorate the edge of the pit, a few layers higher than the surrounding terrace.

The quantitative evaluation of the nonstoichiometry within regime $\mathrm{B}$ follows the same procedure as for regime $\mathrm{A}$, that is, by relating the change in the surface structure to the composition change via the phase diagram (Fig. 1). This means evaluating the relative number of different $(n \times 1)$ lines, as well as accounting for the new $(2 \times 4)$ areas. It is surprising that the stoichiometry of the films of regime $B$ is close to the one of the corresponding substrates and that it does not show a significant trend with fluence (Fig. 2). We must mention that we cannot exclude that systematic errors are introduced during the evaluation of the $(2 \times 4)$ patches and pits: The $(2 \times 4)$ patches are very often quite small and sparse, requiring the acquisition of sufficient statistics on rather large-area images, which, on the other hand, makes it hard to discern $(n \times 1)$ from $(2 \times 4)$ areas. Similar problems occur for the evaluation of the pits. As a result, it is possible that slight $\mathrm{Ti}$ enrichments are in fact introduced with increasing fluences, as the increased number of pits would suggest. Nevertheless, the difference between regimes A and B (Fig. 2) is striking; if a Ti excess is introduced at high fluence, it is significantly smaller than the $\mathrm{Sr}$ excess observed at low fluences and with no apparent trend. Since all films exhibit comparable $\mathrm{Sr} 3 d$ to Ti $2 p$ intensity ratios in XPS (not shown), as well as no statistically significant difference with respect to the $\mathrm{SrTiO}_{3}$ substrates [20], we can exclude that $\mathrm{Ti}$ atoms are implanted in the bulk during Ti-rich growth; the simplest explanation for achieving quasi-ideal stoichiometries with a Ti-rich flux is that
$\mathrm{TiO}_{x}$ species stick less on the newly formed Ti-rich surfaces than $\mathrm{SrO}_{x}$ species.

It is interesting to observe that a qualitative analysis of the RHEED oscillations can be misleading in predicting the evolution of the film morphologies during the growth. In Figs. 3(e) and 3(f) we show the RHEED oscillations of two representative $\mathrm{Sr}$-rich and Ti-rich films, corresponding to the films in Figs. 3( $\left.a_{1}\right)$ and $3\left(c_{1}\right)$, respectively. Generally, a decrease in the intensity of maxima in the RHEED oscillations is assigned to surface roughening. However, this decrease is present in both regime A, Sr-rich growth [Fig. 3(e)], and regime B [Fig. 3(f)], although an appreciable surface roughening only occurs in the latter case. We note that the intensity of oscillation minima of Sr-rich growths increases as the growth proceeds [Fig. 3(e)], while staying roughly constant in the Ti-rich regime. It is plausible that the diffraction geometry, initially optimized for the $(4 \times 1)$ reconstruction, is not ideal for the developing $(5 \times 1)$ and $(6 \times 1)$ phases, causing an overall decrease of the contrast in RHEED oscillations. Interestingly, this effect is markedly pronounced when moving between reconstructions belonging to the same $(n \times 1)$ family. We conclude that the RHEED oscillations are not necessarily a reliable means to predict the development of the film morphology and that the diffraction contrast between evolving surface reconstructions should be also taken into account when striving for ideal flat films.

\section{Role of surface reconstruction}

To understand the mechanisms behind the development of rough surfaces in the presence of the $(2 \times 4)$ structure [opposed to the flatness of the films exhibiting uniform $(n \times 1)$ reconstructed surfaces], we investigate in depth the role of the surface reconstruction during the growth of $\mathrm{SrTiO}_{3}(110)$. We do this by evaluating the early stages of growth under different conditions and by starting from different surface reconstructions.

Submonolayer deposition of $\mathrm{SrTiO}_{3}$ on an $(n \times 1)$ reconstructed surface results in a morphology similar to the one shown in Fig. 4 over a wide range of deposition conditions (fluence between 1.3 and $3.9 \mathrm{~J} / \mathrm{cm}^{2}$, pressure between $5 \times 10^{-6}$ and $3 \times 10^{-2}$ mbar $\mathrm{O}_{2}$, and temperature between $650^{\circ} \mathrm{C}$ and $850^{\circ} \mathrm{C}$ ). In each case, the surface is characterized by rectangular, single-layer-thick islands, elongated along the [110] direction. Larger islands are formed when the substrate is hotter, as surface diffusion is enhanced. The islands display the same $(n \times 1)$ reconstruction of the substrate, with the characteristic dark and bright features that appear in correspondence to antiphase domain boundaries [39]; dark dots correspond to vacancy clusters that relieve the strain between the bulk-truncated $\mathrm{SrTiO}_{3}(110)$ and the "ideal" reconstruction layer without antiphase domain boundaries; bright dots are $\mathrm{Sr}^{2+}$ adatoms that restore the charge balance in the surface layer. These features run uninterrupted across the island edges, indicating that the reconstruction floats to the surface during growth [20]. This is enabled by the intrinsic openness and flexibility of the $(n \times 1)$ structure: Its $\mathrm{TiO}_{4}$ tetrahedra can temporarily distort and change their coordination to host additional species [35], thus enabling the flow of material to 

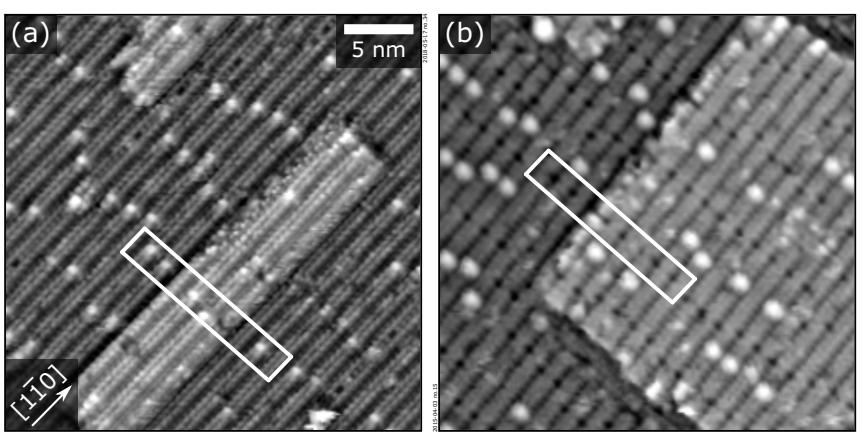

FIG. 4. Submonolayer growth of homoepitaxial $\mathrm{SrTiO}_{3}(110)$ starting from $(n \times 1)$ surfaces. Submonolayer amounts of material deposited on a $(4 \times 1) /(5 \times 1)$-reconstructed surface result in singlelayer-high islands elongated along the [1 $1 \overline{1} 0]$ direction under different growth conditions: (a) four laser pulses, $6 \times 10^{-6}$ mbar $\mathrm{O}_{2}, 750^{\circ} \mathrm{C}$, and $1.7 \mathrm{~J} / \mathrm{cm}^{2}$ and (b) one laser pulse, $3 \times 10^{-2} \mathrm{mbar}_{2}, 850^{\circ} \mathrm{C}$, and $2.0 \mathrm{~J} / \mathrm{cm}^{2}$. The surface reconstruction floats on top of the growing islands that retain the same $(4 \times 1) /(5 \times 1)$ reconstruction as the substrate. Bright and dark dots are an integral part of the surface reconstruction and appear at specific locations on the surface [39]. They form lines that run uninterrupted across the edges of the islands and are highlighted by rectangles. The STM parameters are (a) $V_{\mathrm{b}}=+1.0 \mathrm{~V}$ and $I_{\mathrm{t}}=40 \mathrm{pA}$ and (b) $V_{\mathrm{b}}=+3.3 \mathrm{~V}$ and $I_{\mathrm{t}}=710 \mathrm{pA}$.

subsurface regions, and then easily readjust to the thermodynamically preferred structure.

When starting from a different surface reconstruction, both the island shape and their areal coverage change visibly. Figure 5 compares the islands formed upon submonolayer deposition at the same conditions on different surfaces: monophase $(4 \times 1)$ [Fig. 5(a)], monophase $(2 \times 4)$ [Fig. 5(b)], and mixed, with discrete patches either $(4 \times 1)$ or $(2 \times 4)$ reconstructed [Fig. 5(c)]. The islands are rectangular on both surfaces, but oriented differently: On $(4 \times 1)$ [Fig. $5(\mathrm{a})]$, the preferential direction is [110] (as in Fig. 4), while on the $(2 \times 4)$-reconstructed surface [Fig. 5(b)], islands are elongated along [001]. The island directionality is likely caused by the structural details of the reconstructions which enforce anisotropic diffusion of the adspecies along specific crystallographic directions. The differences in the shapes of the evolving islands are also apparent on the mixed surface [Fig. 5(c)], where the island morphology in each patch is determined by the surface reconstruction of the pristine substrate. Similar islands (aspect ratio and directionality) to those on the monophase $(4 \times 1)$ and $(2 \times 4)$ samples are formed on the $(4 \times 1)$ and $(2 \times 4)$ patches, respectively. Importantly, not only the island shapes, but also their areal coverage is markedly different on the two reconstructions. A statistical analysis reveals that the island coverage changes from $0.237 \pm 0.012$ to $0.144 \pm 0.010$ from $(4 \times 1)$ to $(2 \times 4)$, roughly corresponding to a 1.6-times-smaller areal coverage on $(2 \times 4)$. On the mixed surface, a tendency becomes apparent to accumulate material at the interface between two reconstructions, specifically on the $(4 \times 1)$ side.

We can exclude that the lower coverage on the $(2 \times 4)$ surface is due to step flow: In Fig. 5(b), the island coverage close to the step (20\%, small rectangle) is only slightly larger than in the middle of the terrace (14.4\%, large rectangle); consequently, the lower island coverage on $(2 \times 4)$ areas cannot be assigned to material accumulating at step edges. We explain the differences in island coverage by invoking a different sticking on each surface reconstruction, which is related to their propensity to incorporate the incoming species. Crucial hints come from the details of the surface structures, as have
4 Pulses on a $(4 \times 1)$ Surface

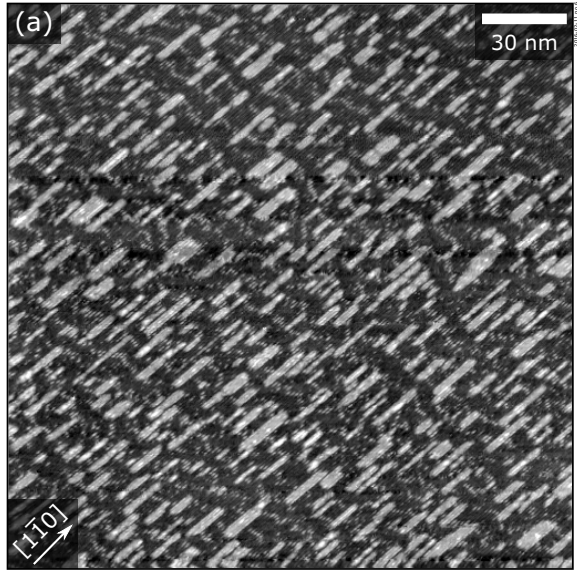

4 Pulses on a $(2 \times 4)$ Surface

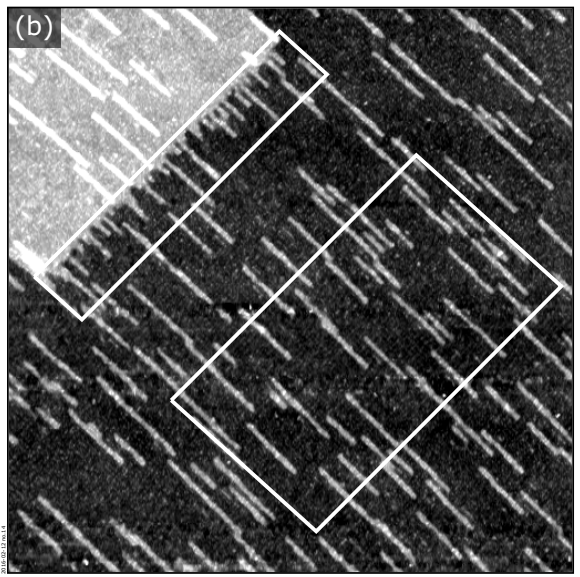

4 Pulses on a Mixed $(4 \times 1) /(2 \times 4)$ Surface

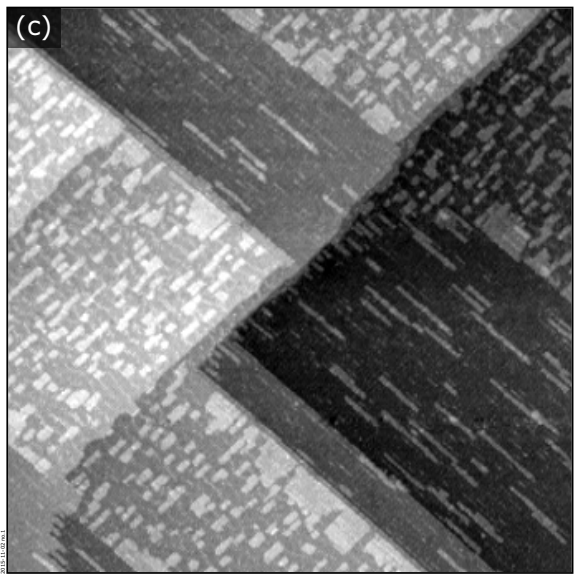

FIG. 5. Surface-dependent sticking on $\mathrm{SrTiO}_{3}(110)$. The same amount of material (four laser pulses at $1 \mathrm{~Hz}, 5 \times 10^{-6} \mathrm{mbar} \mathrm{O}_{2}, 650^{\circ} \mathrm{C}$,

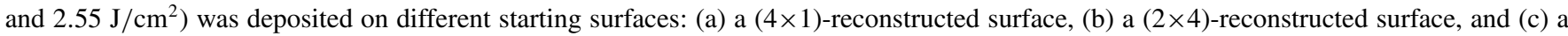
mixed $(4 \times 1) /(2 \times 4)$ surface. Notice that growth on $(4 \times 1)$ [panel (a)] results in the deposition of 1.6 times more material than when starting from a $(2 \times 4)$-reconstructed surface [as in panel (b)]. Also, on the $(4 \times 1)$ surface of panel (a) diffusion is preferential along the [1 10 ] direction, whereas on the $(2 \times 4)$ surface in panel (b) diffusion is faster along the [001] direction. The difference in sticking and diffusion direction is also evident when starting from a mixed $(4 \times 1) /(2 \times 4)$ surface, as shown in panel (c). In panel (b), a small (large) rectangle indicates the area close to (far from) a step, on which fractional island coverage can be evaluated. The STM parameters are (a) $V_{\mathrm{b}}=+1.8 \mathrm{~V}$ and $I_{\mathrm{t}}=290 \mathrm{pA}$, (b) $V_{\mathrm{b}}=+1.8 \mathrm{~V}$ and $I_{\mathrm{t}}=240 \mathrm{pA}$, and (c) $V_{\mathrm{b}}=+2.2 \mathrm{~V}$ and $I_{\mathrm{t}}=360 \mathrm{pA}$. 
also been revealed by recent works that have addressed why $(n \times 1)$ structures are able to incorporate more oxygen from the gas phase than $(l \times m)$ structures [35]. The $(n \times 1)$ structures are open, flexible, and composed of undercoordinated $\mathrm{TiO}_{4}$ tetrahedra, whereas the $(l \times m)$ structures are rigidly packed with fully coordinated $\mathrm{TiO}_{6}$ octahedra. We have recently shown that the $(n \times 1)$ surfaces incorporate more oxygen because their tetrahedra are able to temporarily distort and change their coordination to accommodate oxygen, while this process is highly unfavorable for the saturated octahedra on $(l \times m)$-reconstructed surfaces [35]. A similar mechanism is expected to occur during the growth of $\mathrm{SrTiO}_{3}$. It is less favorable for $(2 \times 4)$ to change the coordination of its stiff and saturated $\mathrm{TiO}_{6}$ octahedra to accommodate the incoming species. Consequently, a higher reevaporation rate is expected on $(2 \times 4)$, which could explain the smaller island coverage measured on this reconstruction. The accumulation of material on $(4 \times 1)$ areas near the boundary of the two reconstructions on the mixed surface [Fig. 5(c)] can be explained with the same idea: Diffusing adatoms on the $(4 \times 1)$ find a high Ehrlich-Schwoebel barrier to traverse the boundary between the two phases, as incorporation in the $(2 \times 4)$ is unfavorable; they are rather "reflected" at the interface between the two phases, while material from the $(2 \times 4)$ side can reach the neighboring $(4 \times 1)$ area, and get accommodated there. Notice that the size of the accumulated material is comparable to that of the islands on the $(4 \times 1)$ terraces, as expected from the diffusion length in that area.

The above considerations allow us to explain the formation of pits in regime B (as defined in Fig. 2): Under (slightly) Ti-rich deposition conditions, $(2 \times 4)$ patches nucleate after the growth of a few layers [Figs. 3( $\left.c_{1}\right)$ and $3\left(c_{2}\right)$ ], perhaps triggered by local fluctuations in the surface composition. As the growth proceeds, due to the smaller sticking on $(2 \times 4)$ areas, the effective growth rate in these regions is slower than on the surrounding terraces and in turn pits develop. Additionally, given the small width of the pits as compared to the distance between islands formed on the $(2 \times 4)$ reconstruction, it is possible that the (fewer) incoming species that stick on the $(2 \times 4)$ patches do not nucleate islands: They could then simply diffuse toward the neighboring $(4 \times 1)$ terrace where they can be more easily incorporated, thus contributing to the rims of $(n \times 1)$ islands decorating the pit [Figs. $3\left(\mathrm{~d}_{1}\right)$ and $3\left(\mathrm{~d}_{2}\right)$ ]; species diffusing on $(n \times 1)$ terraces that find a high barrier to descend toward the pit can also contribute to the development of the rims. Since the typical size of the rims is comparable to the one of islands nucleating on the lower terraces, we conclude that the formation of these rims is to be ascribed to kinetic limitations at this temperature. To conclusively assert whether all incoming material that sticks on $(2 \times 4)$ areas spills out to form the rims, or if it contributes, at least partially, to the (slow) growth of the pit, one should compare the empty volume of the pit with the "rim volume." This quantitative evaluation is unfortunately hindered by the finite sharpness of STM tips that allows us at best to measure a slope of the pit wall around $30^{\circ}$; as a consequence, we believe that the quantitative evaluation of the volume of pits from STM images is unreliable. The saturation of the surface composition with increasing laser fluence (Fig. 2) indicates, however, that Ti-rich species do not stick to, or evaporate from $(2 \times 4)$ areas. What is beyond doubt is that the difference in the accommodating properties between $(2 \times 4)$ and $(n \times 1)$ is responsible for the surface roughening observed in our $\mathrm{SrTiO}_{3}(110)$ films.

\section{CONCLUSION}

We have addressed the origin of surface roughening in PLD-grown $\mathrm{SrTiO}_{3}(110)$ films by investigating the correlation between nonstoichiometries (tuned with the laser fluence), surface structures, and film morphology by means of atomically resolved STM. We have observed that Ti-rich deposition fluxes (obtained at high fluence) trigger the formation of a Tirich $(2 \times 4)$ phase, which shows a smaller sticking coefficient than the coexisting Sr-richer structures. Pits are formed on these low-sticking areas, in turn inducing surface roughening. In this regime, the film stoichiometry is also affected by surface sticking, leading to a quasi-ideal composition instead of the expected Ti enrichment. Surface roughening does not occur when the surface is uniformly reconstructed with Sr-richer structures; however, atomically flat surfaces do not necessarily stand for ideal stoichiometries (as evidenced by our Sr-rich films obtained at decreasing fluences). We envision that the often-reported surface roughening of other complex oxides might be similarly related to the atomic details of surfaces, making the use of analytical tools capable of accessing the surface reconstruction of the film crucial to achieve the desired high-quality films.

\section{ACKNOWLEDGMENTS}

The authors gratefully acknowledge financial support from the Vienna Science and Technology Fund (WWTF), the City of Vienna, and Berndorf Privatstiftung through Project No. MA 16-005; the Austrian Science Fund FWF (Grant No. SFB FOXSI F45); and the TU Wien doctoral school TU-D.
[1] Y. J. Shin et al., Interface control of ferroelectricity in an $\mathrm{SrRuO}_{3} / \mathrm{BaTiO}_{3} / \mathrm{SrRuO}_{3}$ capacitor and its critical thickness, Adv. Mater. 29, 1602795 (2017).

[2] P. Chen et al., Binary controls on interfacial magnetism in manganite heterostructures, Adv. Funct. Mater. 28, 1801766 (2018).

[3] Z. Huang, A. Ariando, X. R. Wang, A. Rusydi, J. Chen, H. Yang, and T. Venkatesan, Interface engineering and emergent phenomena in oxide heterostructures, Adv. Mater. 30, 1802439 (2018).
[4] M. Coll et al., Towards oxide electronics: A roadmap, Appl. Surf. Sci. 482, 1 (2019).

[5] F. Miletto Granozio, G. Koster, and G. Rijnders, Functional oxide interfaces, MRS Bull. 38, 1017 (2013).

[6] P. Zubko, S. Gariglio, M. Gabay, P. Ghosez, and J.-M. Triscone, Interface physics in complex oxide heterostructures, Annu. Rev. Condens. Matter Phys. 2, 141 (2011).

[7] J. J. Yang, D. B. Strukov, and D. R. Stewart, Memristive devices for computing, Nat. Nanotechnol. 8, 13 (2013). 
[8] R. Ramesh and N. A. Spaldin, Multiferroics: Progress and prospects in thin films, Nat. Mater. 6, 21 (2007).

[9] Y. W. Yin et al., Enhanced tunneling electroresistance effect due to a ferroelectrically induced phase transition at a magnetic complex oxide interface, Nat. Mater. 12, 397 (2013).

[10] J. Mannhart and D. G. Schlom, Oxide interfaces-An opportunity for electronics, Science 327, 1607 (2010).

[11] A. Ojeda-G.-P., M. Döbeli, and T. Lippert, Influence of plume properties on thin film composition in pulsed laser deposition, Adv. Mater. Interfaces 5, 1701062 (2018).

[12] S. Gerhold, M. Riva, B. Yildiz, M. Schmid, and U. Diebold, Adjusting island density and morphology of the $\mathrm{SrTiO}_{3}(110)-$ $(4 \times 1)$ surface: Pulsed laser deposition combined with scanning tunneling microscopy, Surf. Sci. 651, 76 (2016).

[13] T. Ohnishi, H. Koinuma, and M. Lippmaa, Pulsed laser deposition of oxide thin films, Appl. Surf. Sci. 252, 2466 (2006).

[14] S. Wicklein, A. Sambri, S. Amoruso, X. Wang, R. Bruzzese, A. Koehl, and R. Dittmann, Pulsed laser ablation of complex oxides: The role of congruent ablation and preferential scattering for the film stoichiometry, Appl. Phys. Lett. 101, 131601 (2012).

[15] T. Ohnishi, K. Shibuya, T. Yamamoto, and M. Lippmaa, Defects and transport in complex oxide thin films, J. Appl. Phys. 103, 103703 (2008).

[16] H. Brune, Microscopic view of epitaxial metal growth: Nucleation and aggregation, Surf. Sci. Rep. 31, 125 (1998).

[17] B. Voigtländer, Fundamental processes in $\mathrm{Si} / \mathrm{Si}$ and $\mathrm{Ge} / \mathrm{Si}$ epitaxy studied by scanning tunneling microscopy during growth, Surf. Sci. Rep. 43, 127 (2001).

[18] T. Ohsawa, M. Saito, R. Shimizu, K. Iwaya, S. Shiraki, Y. Ikuhara, and $\mathrm{T}$. Hitosugi, Impact of a surface $\mathrm{TiO}_{2}$ atomic sheet on the electronic transport properties of $\mathrm{LaAlO}_{3} / \mathrm{SrTiO}_{3}$ heterointerfaces, Appl. Phys. Lett. 113, 141602 (2018).

[19] S.-h. Phark, Y. J. Chang, and T. W. Noh, Selective growth of perovskite oxides on $\mathrm{SrTiO}_{3}(001)$ by control of surface reconstructions, Appl. Phys. Lett. 98, 161908 (2011).

[20] M. Riva, G. Franceschi, Q. Lu, M. Schmid, B. Yildiz, and U. Diebold, Pushing the detection of cation nonstoichiometry to the limit, Phys. Rev. Mater. 3, 043802 (2019).

[21] T. Ohsawa, R. Shimizu, K. Iwaya, S. Shiraki, and T. Hitosugi, Negligible Sr segregation on $\mathrm{SrTiO}_{3}(001)-(\sqrt{13} \times \sqrt{13})-R 33.7^{\circ}$ reconstructed surfaces, Appl. Phys. Lett. 108, 161603 (2016).

[22] F. Yang, Y. Liang, L.-X. Liu, Q. Zhu, W.-H. Wang, X.-T. Zhu, and J.-D. Guo, Controlled growth of complex polar oxide films with atomically precise molecular beam epitaxy, Front. Phys. 13, 136802 (2018).

[23] T. K. Andersen, D. D. Fong, and L. D. Marks, Pauling's rules for oxide surfaces, Surf. Sci. Rep. 73, 213 (2018).

[24] S. Cook and L. D. Marks, Ab initio predictions of double-layer $\mathrm{TiO}_{2}$-terminated $\mathrm{SrTiO}_{3}(001)$ surface reconstructions, J. Phys. Chem. C 122, 21991 (2018).
[25] S. Gerhold, Z. Wang, M. Schmid, and U. Diebold, Stoichiometry-driven switching between surface reconstructions on $\mathrm{SrTiO}_{3}(001)$, Surf. Sci. 621, L1 (2014).

[26] B. C. Russell and M. R. Castell, Surface of sputtered and annealed polar $\mathrm{SrTiO}_{3}(111)$ : $\mathrm{TiO}_{x}$-rich $(n \times n)$ reconstructions, J. Phys. Chem. C 112, 6538 (2008).

[27] D. M. Kienzle, A. E. Becerra-Toledo, and L. D. Marks, Vacant-Site Octahedral Tilings on $\mathrm{SrTiO}_{3}(001)$, the $(\sqrt{13} \times \sqrt{13}) R 33.7^{\circ}$ Surface, and Related Structures, Phys. Rev. Lett. 106, 176102 (2011).

[28] J. A. Enterkin, A. K. Subramanian, B. C. Russell, M. R. Castell, K. R. Poeppelmeier, and L. D. Marks, A homologous series of structures on the surface of $\mathrm{SrTiO}_{3}(110)$, Nat. Mater. 9, 245 (2010).

[29] T. Kubo and H. Nozoye, Surface Structure of $\operatorname{SrTiO}_{3}(100)$ $(\sqrt{5} \times \sqrt{5})-R 26.6^{\circ}$, Phys. Rev. Lett. 86, 1801 (2001).

[30] Z. Wang, J. Feng, Y. Yang, Y. Yao, L. Gu, F. Yang, Q. Guo, and J. Guo, Cation stoichiometry optimization of $\mathrm{SrTiO}_{3}(110)$ thin films with atomic precision in homogeneous molecular beam epitaxy, Appl. Phys. Lett. 100, 051602 (2012).

[31] J. Goniakowski, F. Finocchi, and C. Noguera, Polarity of oxide surfaces and nanostructures, Rep. Prog. Phys. 71, 016501 (2008).

[32] Z. Wang et al., Transition from reconstruction toward thin film on the (110) surface of strontium titanate, Nano Lett. 16, 2407 (2016).

[33] B. Dam, J. H. Rector, J. Johansson, J. Huijbregtse, and D. G. De Groot, Mechanism of incongruent ablation of $\mathrm{SrTiO}_{3}$, J. Appl. Phys. 83, 3386 (1998).

[34] R. Meyer, A. F. Zurhelle, R. A. De Souza, R. Waser, and F. Gunkel, Dynamics of the metal-insulator transition of donordoped $\mathrm{SrTiO}_{3}$, Phys. Rev. B 94, 115408 (2016).

[35] M. Riva, M. Kubicek, X. Hao, G. Franceschi, S. Gerhold, M. Schmid, H. Hutter, J. Fleig, C. Franchini, B. Yildiz, and U. Diebold, Influence of surface atomic structure demonstrated on oxygen incorporation mechanism at a model perovskite oxide, Nat. Commun. 9, 3710 (2018).

[36] Z. Wang, F. Yang, Z. Zhang, Y. Tang, J. Feng, K. Wu, Q. Guo, and J. Guo, Evolution of the surface structures on $\mathrm{SrTiO}_{3}(110)$ tuned by Ti or Sr concentration, Phys. Rev. B 83, 155453 (2011).

[37] G. Z. Liu, Q. Y. Lei, and X. X. Xi, Stoichiometry of $\mathrm{SrTiO}_{3}$ films grown by pulsed laser deposition, Appl. Phys. Lett. 100, 202902 (2012).

[38] Z. Wang, Epitaxial growth and investigation of the surface properties of $\mathrm{SrTiO}_{3}$ thin films, Ph.D. thesis, Chinese Academy of Sciences, 2011.

[39] Z. Wang, F. Li, S. Meng, J. Zhang, E. W. Plummer, U. Diebold, and J. Guo, Strain-Induced Defect Superstructure on the $\mathrm{SrTiO}_{3}(110)$ Surface, Phys. Rev. Lett. 111, 056101 (2013). 